\title{
Combined impact of pre-sensitization and delayed graft function on the development of allograft rejection in deceased donor kidney transplantation: nationwide cohort study
}

\author{
Hanbi Lee ${ }^{1}$, Yohan Park ${ }^{1}$, Tae Hyun Ban², Sang Heon Song ${ }^{3}$, Seung Hwan Song ${ }^{4}$, Jaeseok Yang ${ }^{5}$, Curie Ahn ${ }^{5}$, \\ Chul Woo Yang ${ }^{1}$, Byung Ha Chung ${ }^{1}$ \\ 1'Division of Nephrology, Department of Internal Medicine, Seoul St. Mary's Hospital, College of Medicine, The Catholic University of Korea, Seoul, Korea \\ ${ }^{2}$ Division of Nephrology, Department of Internal Medicine, Eunpyeong St. Mary's Hospital, College of Medicine, The Catholic University of Korea, Seoul, Korea \\ ${ }^{3}$ Division of Nephrology, Department of Internal Medicine, Pusan National University Hospital, Busan, Korea \\ ${ }^{4}$ Department of Surgery, Ewha Womans University Seoul Hospital, Seoul, Korea \\ ${ }^{5}$ Division of Nephrology, Department of Internal Medicine, Seoul National University Hospital, Seoul, Korea
}

Background: Both pre-sensitization to human leukocyte antigen and delayed graft function (DGF) is well documented to be associated with poor allograft outcome. The aim of this study is to investigate whether development of DGF and pre-transplant highly sensitization has synergistic adverse effect on the allograft outcome after deceased donor kidney transplantation (DDKT) using Korean Transplant Registry (KOTRY) database, the nationwide prospective cohort.

Methods: Between May 2014 and June 2019, total 1,945 DDKT were registered in KOTRY database and out of them, 1,370 cases were included in this study. According to pre-sensitization status and development of DGF post-transplant, they were divided into four groups: non-pre-sensitized-DGF(-) $(n=1,100)$, non-pre-sensitized-DGF $(+)(n=133)$, pre-sensitized-DGF $(-)(n=116)$, and pre-sensitized-DGF(+) $(n=21)$. We compared allograft rejection, the change of allograft function, allograft survival, patient survival, and post-transplant complications across four groups.

Results: The incidence of overall biopsy-proven rejection and acute antibody-mediated rejection (ABMR) was significantly higher in the pre-sensitized-DGF(+) group than the other three groups. In addition, multivariable analysis demonstrated that pre-sensitization combined with DGF is an independent risk factor for both overall rejection and ABMR compared to non-pre-sensitizedDGF(-) group (hazard ratio, 10.769; 95\% confidence interval, 3.717-31.198; $\mathrm{P}<0.001$ ). Moreover, DGF and pre-sensitization showed statistically significant interaction each other ( $P$ for interaction $<0.001)$. Pre-sensitization combined with DGF did not show a significant impact on allograft function and allograft or patient survival in this study.

Conclusions: In conclusion, pre-sensitization and DGF show a synergistic adverse impact on the post-transplant allograft outcome in terms of allograft rejection after DDKT. Therefore, we suggest when DGF occurred in DDKT with pre-sensitization, more careful monitoring or surveillance for allograft rejection is required.

Corresponding author: Byung Ha Chung

E-mail: chungbh@catholic.ac.kr

This is an Open Access article distributed under the terms of the Creative Commons Attribution Non-Commercial License (http://creativecommons.org/licenses/by-nc/4.0/) which permits unrestricted non-commercial use, distribution, and reproduction in any medium, provided the original work is properly cited. 